\title{
KNOWLEDGE AND SKILLS CHANGES TO ACCREDITATION HERALD PEDAGOGICAL TRANSFORMATION IN THE UK
}

\author{
I David Sands - University of Hull - DOI: https://doi.org/10.1051/epn/2019505
}

The Institute of Physics in London is changing the way it accredits degrees, which could have far-reaching consequences for the way physics is taught and assessed. Degree accreditation serves two purposes. First, it is the mechanism by which the Institute fulfils its commitment under its Royal Charter to uphold standards in physics education, and secondly, it provides a crucial step toward professional recognition for graduates.
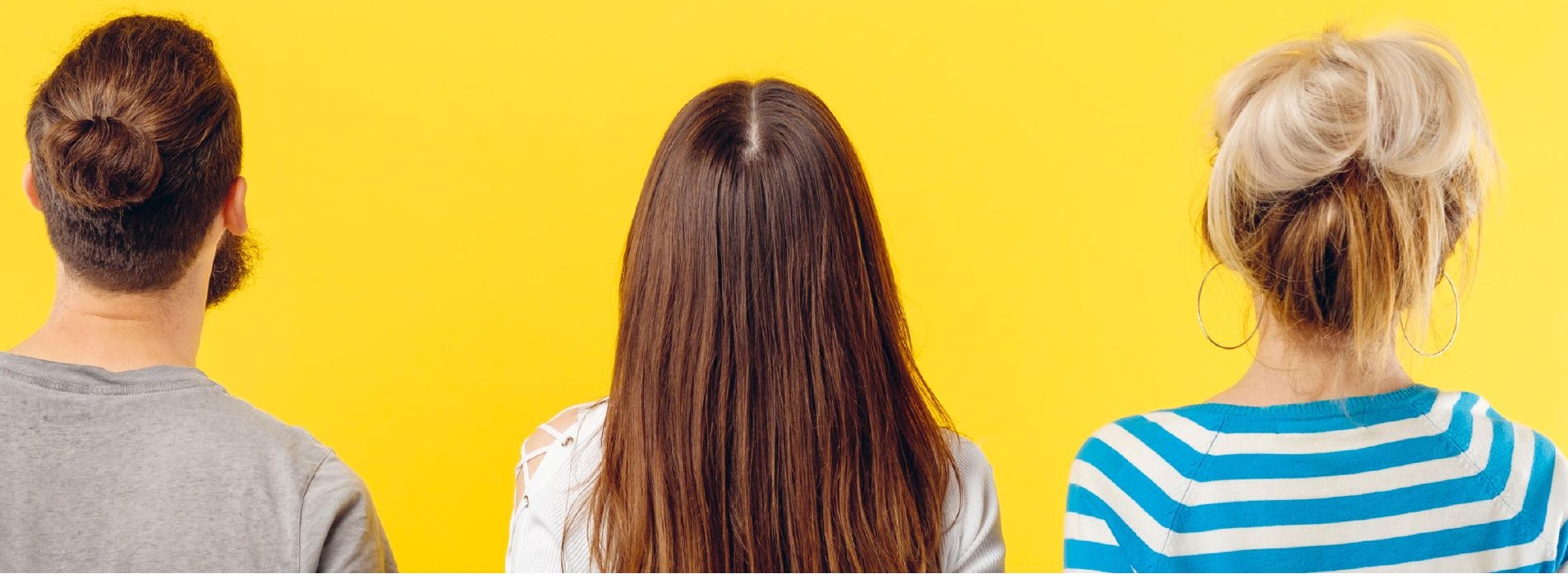

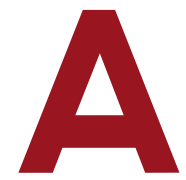

nyone wanting to be recognised as a Chartered Physicist has not only to be able to provide evidence of suitable professional experience, but also to show knowledge and skills appropriate to Masters level. By accrediting degree programmes, the Institute makes it much easier for graduates to be able to demonstrate the requisite educational level or knowledge level has been reached.

Those familiar with the UK system will be aware that we have two types of undergraduate degrees: the normal 3-year Bachelors and the 4-year integrated Masters. The Institute accredits both types of degree. Graduates from an accredited Bachelors degree partially meet the educational requirements for professional recognition, but graduates from an integrated Masters degree meet them in full and only have to show appropriate professional experience.

The Institute has previously approached accreditation by essentially defining what a physics degree should look like. There is a prescribed core of topics together with some attempt to define the minimum level of complexity.
Referred to as the IOP Core of Physics, this content is largely delivered in the first two years. In addition to the Core, a Bachelors programme must contain at least 60 credits (CATS) of honours-level physics content. For those not familiar with the British system, honours level corresponds to the final level of a Bachelors programme. There are also suggested minimum amounts of laboratory work for experimental physics programmes, as well as a range of skills that graduates should be developing and requirements on a minimum amount of mathematics content.

What often seems like a good idea in principle can throw up difficulties in practice and there are three main drawbacks to the current approach of requiring a fairly substantial prescribed core content. First, physics degrees across the UK look remarkably similar. There are variations, of course, but as the first two years of any Bachelors degree are generally taken up with teaching the IOP Core the opportunities to be distinctive are limited to what is offered in the final year. Secondly, rather than being seen as the essential physics that every graduate should know, the Core of Physics has come to be seen 
as a requirement to be fulfilled and very often depth is sacrificed for breadth with some material being covered in only one or two lectures. Thirdly, the kinds of physics degrees that can meet the accreditation requirements are quite limited.

This last is a central consideration in the accreditation review. The Core contains a number of what seem at first sight to be important concepts but in fact are very specific examples of the application of more basic ideas. In degree programmes such as biological physics or environmental physics, which are concerned with the application of physics to specific areas, it might not be relevant for students to learn in depth about laser cavities, semiconductor band structure, or the role of phonons in the heat capacity of a solid. That does not mean that they do not possess a sound knowledge of physics, think like physicists or acquire the same kind of physics-related skills as graduates from more conventional physics degrees. If these kinds of degrees deliver these outcomes, graduates should be eligible for the same kind of accelerated professional recognition.

The answer to these difficulties is to shift the focus away from the degree to the graduate. The existing accreditation scheme sets out in detail what a physics degree should like, or at least those essential elements common to physics degrees across the UK, but we want instead to identify the kind of attributes a graduate should possess. It will be up to departments to decide on the details of the degree programme that will develop those attributes and this will allow for much greater flexibility, distinctiveness and inclusivity.

Our starting point for graduate attributes are the QAA benchmark statements for physics and astronomy ${ }^{1}$. At the end of the last century the QAA, or Quality Assurance Agency, started to lay down a set of discipline-specific statements against which the outcomes of degrees should be judged. These statements were constructed by the members of the respective academic communities, for example, lawyers, historians, mathematicians, etc., and therefore reflected the thinking of that community. Regardless of the university or the discipline, it is now very unlikely that any new degree will not base its outcomes on the benchmark statements. These statements have thus become the de facto standards for the outcomes of degree programmes in the UK.

The last revision of the benchmark statements for physics and astronomoccurred in 2017. I was part of the review group. We came to the conclusion early on that little change was needed to the content, but it could be re-ordered and re-organized to present a much more coherent and usable account of standards. Thus, the 2017 document looks very different from its 2008 predecessor, but the changes are largely cosmetic. The long list of outcomes for both Bachelors and Masters degrees was reorganised into threshold and typical outcomes for both types of degree. The typical outcomes for a BSc build on the threshold and the threshold for integrated Masters degrees build on the typical for a Bachelors. The only change of any substance we made was to emphasise the role of computation in modern physics, which was understated in the 2008 document. Computational physics has emerged as a third way of doing physics alongside the two traditional branches of experimental and theoretical physics. In particular, computation is perhaps the only possible way to understand emergent behaviour in large systems subject to simple rules and we wanted the benchmark statement to reflect this.

Although we weren't thinking about accreditation at the time, re-organising the benchmark statements into threshold and typical levels brought the two processes into very close alignment. Accreditation is very much concerned with thresholds: every graduate from a degree programme must meet the minimum educational standard for that programme to be accredited. Therefore, we adopted and adapted the outcomes in the benchmark statement for our own purposes.

The principal adaptation has been to phrase the outcomes in a way that is clear and measurable. There are eight threshold standards for a Bachelor's degree and most can be carried across into accreditation without much adjustment or elaboration, but some are not so straightforward. For example, the first standard says that a student will have demonstrated an ability to "comprehend basic physical laws and principles", which begs two questions: what do we mean by comprehend and which basic laws?

Understanding is one of those things that we recognise when we see it but is very hard to pin down in a simple definition, yet if a department is to collect evidence that students are developing an understanding and present that in support of an accreditation application, this is precisely what must be done. Physics education research has shown repeatedly over the years that being able to state a law is not equivalent to understanding it. Students can often state Newton's three laws of motion, for example,

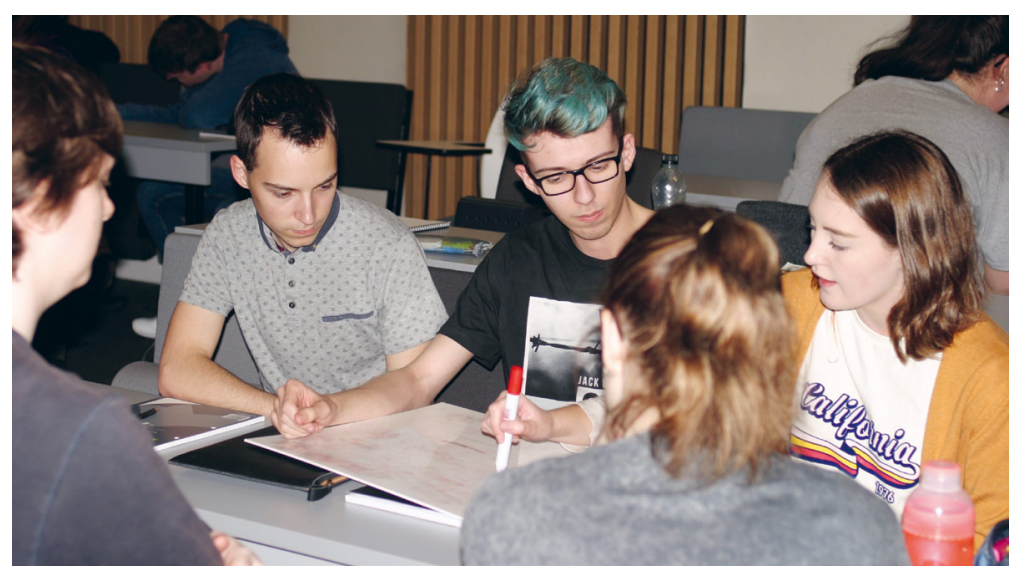

${ }^{1}$ https://www.qaa.ac.uk/docs/qaa/subject-benchmark-statements/sbs-physics-astronomy-and-astrophysics-17.pdf 


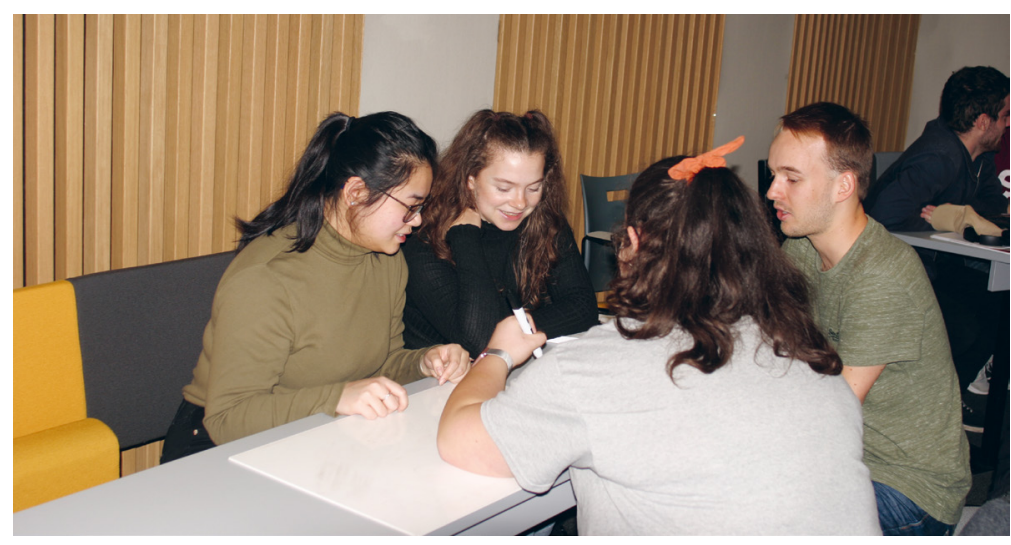

but when asked to apply them in a relatively simple problem requiring qualitative reasoning, they often come up with the wrong answer. However, skill in mathematics is also not sufficient. Graduates who demonstrate mathematical facility do not always display a good grasp of the fundamentals.

If you were to have an intelligent conversation with someone about physics, you would expect the conversation to involve qualitative descriptions as well as qualitative and mathematical arguments. The last might involve, for example, limiting cases or simply interpreting trends implied by mathematical representations of the physics. You would also expect ideas to be represented in whatever way is appropriate to the discussion, including using diagrams and graphs. The ability to reason qualitatively and to translate between different representations lies at the heart of understanding and in the absence of a precise definition these will serve as good indicators. Therefore, by including understanding of basic laws and principles among the accreditation criteria we will effectively require departments to find ways to allow students to develop these abilities as well as assess them and to use both as evidence in their accreditation submission.

The question of what to include in the content is more difficult to resolve. If you were to ask your colleagues which ideas in physics a graduate must know in order to be considered a physicist, you would probably receive a variety of different answers depending on the particular specialism of the person you are asking. By way of example, a colleague of mine suggested not so long ago that the $\mathrm{p}$-n junction is so central to modern technology that it ought to feature in the IOP core. It doesn't and never has, but one can easily appreciate the argument for including it. It functions as a rectifier and a voltage-dependent capacitor, but it also forms the basis of laser diodes, LEDs, solar cells, transistors and photodiodes. However, these are applications of semiconductor band theory, which in turn is arises from the application of the Pauli exclusion principle to interacting systems. If we were to pare it down to essentials, we would find that semiconductor physics is an application of quantum mechanics to interacting, multi-atom and multi-electron systems with some simplifying assumptions from classical mechanics (in the form of transport theory) and electrostatics superimposed. It might be desirable to teach these ideas via semiconductor physics, but it is not essential. What is essential, however, is that students have the skills for self-learning, so that someone who has learnt these ideas in other contexts can, if necessary, transfer them to semiconductors. The same applies to many of the ideas that would be suggested for inclusion in the core.

We have taken a quite radical approach to the IOP Core of Physics. Our intention at the outset was to make it less prescriptive and restrictive, so we have based it on the areas identified as fundamental in the benchmark statement: electromagnetism, quantum and classical mechanics, statistical physics and thermodynamics, wave phenomena and the properties of matter. Two topics currently in the Core, condensed matter and optics, no longer feature in the requirements for accreditation. We reasoned that a graduate with a sound understanding of these five basic topics should be able to pick up optics or solid state physics through self-directed learning if the need arises. Most departments are likely to continue teaching these topics but removing them from the core gives more freedom to consider whether this content is necessary for all degrees, for example mathematical physics. If taught, they would then serve as vehicles to illustrate the application of some or all of these five fundamental topics.

The main outcome of the review of accreditation procedures is that the IOP Core of Physics is no longer a list of topics that needs to be taught in the first two years. Rather, it constitutes a set of themes that will run through the entire degree and in a well-designed programme students will develop their knowledge, understanding and competence in applying that knowledge throughout the duration of the degree. This allows for a much more holistic approach to the design of the curriculum and has the additional benefit that departments can be much more distinctive in what they offer. Considerable support for these ideas has already been expressed but a formal consultation is required before the scheme is implemented. Once that happens, either towards the end of this year or early next, it will take time for departments to adjust to the new scheme, but it holds out the prospect of systematically embedding good teaching practice into the national structure of physics degrees in the UK. -

\section{About the Author}

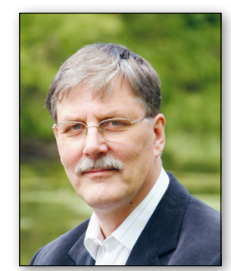

David Sands is chair of the Physics Education Division of the EPS. He has worked extensively with the Institute of Physics in London on accreditation, first as an assessor, then as chair of the Degree Accreditation Committee and lastly as chair of the Accreditation Review Group. He also represents the UK on Commission 14 (education) of IUPAP. 